\title{
Study of Soil Microbial Biomass and Enzymatic Activity
}

\author{
Jingjing Peng \\ Taishan University, Tai'an, Shandong, 271000, China \\ Shandong Agricultural University, Tai'an, Shandong, 271000, China
}

\begin{abstract}
Keywords: Hulun Buir Grassland; microbial biomass; enzymatic activity; research.
\end{abstract}
\begin{abstract}
Taking five different grassland types of Hulun Buir Grassland (the meadowsweet plain, stipa baicalensis plain, guinea grass plain, stipa grandis plain and stipa krylovii plain) as the research objects, this paper respectively studied the change characteristics of their soil physicochemical properties, soil microbial quantity, soil microbial biomass and soil enzymatic activity as well as their relationship with each other. The results showed that there is a correlation between the soil microbe quantity, soil microbial biomass, the enzymatic activity and the physicochemical properties in different grassland types on Hulun Buir Grassland. It can be concluded from the analysis that, the soil microbial biomass carbon increases with the increase of the water content and bacteria in the soil and it decreases with the increase of phosphatase activity in soil; the soil microbial biomass nitrogen increases with the increase of soil catalase activity and decreases with the increase of soil phosphatase activity and soil bulk density.
\end{abstract}

\section{Introduction}

Hulun Buir Grassland is one of the most typical temperate grasslands in China. It is located in the eastern part of the Inner Mongolia Plateau, at $115^{\circ} \sim 121^{\circ}$ east longitude and $47^{\circ} \sim 50^{\circ}$ north latitude. It is a very important part of the Eurasian steppe. The total area of Hulun Buir Grassland is about $7,853,200 \mathrm{hm} 2$. The plain types of Hulun Buir Grassland are very complex and diverse. The area of the temperate meadow grassland is about $1,419,000 \mathrm{hm} 2$, accounting for $18.07 \%$ of the total area of Hulun Buir Grassland. The main plain types of the temperate meadow steppe include meadowsweet grassland, stipa baicalensis plain, etc. There are also large warm typical grasslands the area of which is about $4,314,100 \mathrm{hm} 2$, accounting for $54.93 \%$ of the total area of the grassland. The main plain types of the temperate typical grassland include the guinea grass plain, stipa grandis plain and stipa krylovii plain. As a world-famous research base of grassland resources and base of biodiversity conservation, Hulun Buir Grassland has always been highly valued and focused by well-known scholars at home and abroad. Therefore, to strengthen the study and research on the ecological environment system in Hulun Buir Grassland not only has great guiding significance for its own biodiversity conservation and sustainable development, but also helps to the exploration and study of ecological environment systems of temperate grasslands in China and even the whole world, which will contribute to the development and progress of ecology.

Soil microbes constitute a very important part of the ecosystem and have a very significant influence on the material circulation and energy conversion in the grassland ecosystem. Although the content of soil microbes occupies only a very small part of the soil nutrient pool, soil microbes are not only the "reservoir" but also the "source" of soil nutrients.[1] Especially in the evolution of grassland soil quality, soil microbes not only have a very high ability to convert nutrients, but also are involved in the energy flow and material flow in ecosystems, affecting the plant nutrition, soil structure and soil fertility and other aspects in grassland ecosystems; at the same time, soil microbial biomass is extremely sensitive to environmental changes and can indicate specific changes of ecosystem functions, and therefore, it is one of the important parameters to evaluate the environmental quality of grassland soil. The soil enzyme is mainly derived from the secretions and residues of animals and plants, microbial secretions, etc. It participates in the chemical processes of all organisms in the soil and its activity represents the level of material metabolism in the soil. Soil enzymes and microbes 
promote the biochemical process of grass soil. At the same time, the soil enzyme also plays a very important role in the process of material conversion and has a very important influence on the evolution of soil fertility. Different grassland types of the Hulun Buir Grassland were selected as the research objects in this research to study their respective main soil physicochemical properties, soil microbial flora, soil microbial quantity, soil enzymatic activity as well as their relationship with each other, hoping to offer the basic data for the study of the structure and function of Hulun Buir Grassland's ecosystem and also provide a theoretical basis for the sustainable development of different types of grasslands.

\section{Materials and Methods}

\subsection{Overview of The Research Area}

The experimental plots were in the territory of Hulun Buir in Inner Mongolia. They located at $47^{\circ} \sim$ $53^{\circ}$ north latitude and $115^{\circ} \sim 126^{\circ}$ east longitude. The area belongs to the typical temperate continental monsoon climate, with an annual average temperature of $-2.2^{\circ} \mathrm{C}$. There is no frost in this area and the annual rainfall is $339 \mathrm{~mm}$. The main research objects are the meadow grasslands (meadowsweet plain and stipa baicalensis plain) and typical grasslands (guinea grass plain, stipa grandis plain and stipa krylovii plain) on Hulun Buir Grassland. In the open areas with the typical vegetation and relatively flat terrain, grasslands fenced since 2008 were respectively selected as the sample plots and the enclosure lasted 8 years.

\subsection{Soil Sampling and Pretreatment}

The field sampling was conducted in July 2009. Each sampling site set 20 random sampling points and the spacing between sampling points were more than $100 \mathrm{~m}$.[1] The boring stick with a diameter of about $9 \mathrm{~cm}$ was employed for sampling and the soil respectively at $0 \sim 10$ and $10 \sim 20 \mathrm{~cm}$ in the soil as was taken as the samples. Soil wrapped with fresh bags was taken back to the laboratory and then plant roots and gravel were removed. After being layering evenly, each soil sample was divided into two equal parts. The first part was saved in the refrigerator at $4{ }^{\circ} \mathrm{C}$ and it was used for the determination of soil microbial quantity and soil microbial biomass. The second part was air-dried indoors. After the air-dried soil was sifted with 0.15 and $1.00 \mathrm{~mm}$ griddle, it was used for the study and determination of physicochemical properties and enzymatic activity of soil.

\subsection{Determination and Analysis Methods}

Quantification of microbes :plate count was adopted; bacteria used beef peptone agar culture; fungus used rose-bengal agar culture; and actinomycetes adopted modified Gause I medium, the incubation time was $2 \sim 5$ d.Determination of soil enzymatic activity: soil urease activity was determined by indophenol blue colorimetric assay, and its activity was expressed as milligrams of $\mathrm{NH}+-\mathrm{N}$ per gram of air-dried soil after culturing for $24 \mathrm{~h}$.Determination of soil physicochemical properties: conventional analysis was adopted.PH value adopted acidimetric method; the soil moisture content was measured by oven drying method; soil bulk density adopted by cutting-ring method. [3]

\section{Results and Analysis}

\subsection{Soil Physicochemical Properties of Different Types of Grasslands}

Soil physicochemical properties of different grassland types are significantly different (P $<0.05)$. The $\mathrm{pH}$ value of soil in the meadowsweet plain is low; its soil bulk density is small, but it has the highest soil moisture and soil nutrient content. The $\mathrm{pH}$ value of soil in the the guinea grass plain is high; its soil bulk density is small; and the soil moisture and soil nutrient content is high. The $\mathrm{pH}$ value of soil in the stipa grandis plain is high; its soil bulk density is big; and it has the lowest soil moisture and soil nutrient content. Therefore, the meadowsweet plain is the most fertile in the five types of grasslands while the stipa grandis plain is the poorest. 


\subsection{Soil Microbial Composition of Different Grassland Types}

According to the survey, the quantity and composition of microbes in the soil of different types of grasslands on Hulun Buir Grassland are not the same. Therefore, it can be concluded that the type and quantity of microbes are affected by the environment. The study showed that, in the soil of five grasslands studied, bacteria are the most numerous, followed by actinomycetes; and fungi is the least numerous. The amount of bacteria is also different in the soil of different grassland types. The guinea grass plain holds the most bacteria, respectively followed by the stipa baicalensis plain, stipa krylovii plain and then Meadowsweet plain; the amount of bacteria in the soil of the stipa grandis plain is the least. The proportions of actinomycetes from the high to low in the soil of different types of grasslands are respectively the stipa krylovii plain, guinea grass plain, meadowsweet plain, stipa baicalensis plain and finally the stipa grandis plain. The proportions of fungus in the soil of different types of grasslands are: the meadowsweet plain>stipa krylovii plain>guinea grass plain>stipa baicalensis plain>stipa grandis plain.

\subsection{Soil Enzymatic Activity of Different Types of Grasslands}

The research shows that the activity of soil catalase, invertase, urease and phosphatase is different in different grassland types. Firstly, the enzymatic activity in the surface soil is generally higher than that in the lower soil layer. Secondly, the catalase showed the highest activity in the soil of the meadowsweet plain, and the enzymatic activity in the soil of the other four grasslands is also different.

\section{Discussion}

\subsection{Distribution Characteristics of Microbial Quantity and Soil Microbial Biomass in the Soil of Different Grassland Types}

Soil microbes play a very significant role in the grassland ecosystem and are an integral part in the system of "soil -- soil microbes -- plant". They have a very crucial influence on the nutrients, structural composition and ecological stability of the soil as well as the ecological restoration of the grassland vegetation. [4]Although soil microbes only account for a very small proportion of organic matter, they are the most active components of organic matter, and they are the most active part especially in the carbon and nitrogen cycling process. It can be seen from the results above that, the distribution characteristics of bacteria, actinomycetes and fungi in soil of the five different types of grasslands on Hulun Buir Grassland have the following relation: the amount of bacteria is larger than that of actinomycetes and the actinomycetes are more than that of fungi. This conclusion is similar to that of most of the scholars surveyed. The soil of different grassland types on the Hulun Buir Grassland has different temperature and humidity because of the different types of vegetation composition and different degrees of coverage on the soil surface. In addition, the quantity, size, dry branches and fallen leaves of different plants are not the same in different vegetation community. Due to the influence of many factors above, the amount and the activity status of soil microbes of different grassland types are different.

It can be learned from the investigation that, the quantity of bacteria and fungi in the soil of five different types of grasslands on Hulun Buir Grassland have a positive correlation with the soil nutrient content and soil water content. In other words, the higher the nutrient content of the soil, the higher the amount of microbes in the soil is. This is because the soil fertility, soil environmental conditions and soil microbes cooperate with each other and develop together. Healthy soil environment can promote the reproduction and healthy growth of microbes in the soil. At the same time, the microbes in the soil can in turn provide favorable feedback to the soil structure of the grassland. Nutrients accumulated by microbes and microbial transformation function play a good role in promoting grassland soil structure.

\subsection{Soil Enzymatic Activity Characteristics of Different Grassland Types}

Soil enzyme is one of the most active organic components in soil, which not only helps the soil nutrient circulation, but also provides nutrients needed for the growth and development of grassland plants. According to the research results, the activity of catalase, invertase, urease and phosphatase in 
the soil of different types of grasslands on Hulun Buir Grassland is different. Moreover, the activity of the enzyme in the soil decreases with the depth increase of the soil layer. This conclusion is the same as the research results of Hou Yanhui and other scholars. This conclusion is also explained in this research. The main reason is that, after years of accumulation, the soil is covered by thick humus and a lot of dry branches and fallen leaves, so the organic matter content of the soil surface is relatively high. Humus provides a sufficient source of nutrients to the surface of the soil, which is very beneficial to the growth of microbes in the soil. In addition, the surface of the soil is well ventilated and the water and heat conditions are good. These factors have resulted in the growth of microbes on the soil surface and the active metabolism, thus greatly improving the activity of enzyme in the soil surface.

\subsection{The Relationship Between Microbial Biomass and Other Soil Environmental Factors in Soil}

Soil microbial biomass is one of the most active parts and also one of the most vulnerable parts of the soil .It is easily affected by some soil environmental factors. The content of soil microbes is related to the type of soil, the type of vegetation on the soil and the local climate. The carbon content of soil microbial biomass in the five studied grassland types is affected by the soil moisture, the number of bacteria in soil and the activity of soil phosphatase. What affects the carbon content of microbial biomass is mainly the content of soil moisture, because it influences the development and growth of microbes in the soil.

The nitrogen content of microbes in the soil is easily affected by the content of the soil catalase, activity of soil phosphatas and soil bulk density. The main reason is that the catalase in the soil is secreted by bacteria, fungi and the roots of plants in the soil. The soil catalase can be directly involved in the transformation and energy conversion of all the substances in the soil. Catalase activity can represent the strength of biological oxidation in a certain extent and also reflects the intensity of microbial activity in soil. In addition to directly affecting soil aeration, soil porosity and penetration resistance of the plant root system, soil bulk density can affect the growth and development of plant roots. The catalase in soil can also indirectly influence the biological activity of the soil through the above factors. Therefore, the increase of the soil catalase activity and the decrease of the soil bulk density will all affect the nitrogen content of the soil microbial biomass.

\section{Conclusion}

By studying the soil microbial biomass and enzymatic activity in different grassland types of Hulun Buir Grassland, it can be seen that the microbial biomass and enzymatic activity are different in the soil of different grassland types. It is concluded that, besides the grassland area, the soil microbial biomass and enzymatic activity in many other parts of China are also different from each other, which needs further research.

\section{References}

[1]. Wang Weihua, Liu Yi, Sun Zhilong. The Effect of Long-term Fertilization on Microbial Biomass, Community Structure and Activity in Paddy Soil [J]. Environmental Science, 2018 (01): 1-11 [2018-01-23].

[2]. Tan Hongyan, Yan Ruirui, Yan Yuchun, Chen Baorui, Xin Xiaoping. PLFAs Analysis of Soil Microbial Community Structure of Temperate Meadow Steppe under Different Grazing Intensity [J]. Acta Prataculturae Sinica, 2015, 24 (03): 115-121.

[3]. Gu Xuejin, Zhao Ji, Wang Juan. Microbial Biomass of Soil in the Typical Grassland of Inner Mongolia [J]. Journal of Agro-Environment Science, 2007 (04): 1444-1448.

[4]. Quan Guoling, Xie Kaiyun, Tong Zongyong, Li Xianglin, Wan Liqiang, Bi Shuyi, Wan Xiufu. Effect of Compound Microbial Fertilizer on Enzyme Activity and Physicochemical Properties of Guinea Grass Plain Soil [J]. Acta Prataculturae Sinica, 2016, 25 (02): 27-36. 